CfPA-TH-92-21

July 9, 1992

\title{
COBE's Constraints on the Global Monopole and Texture Theories of Cosmic Structure Formation
}

\author{
DAVid P. BenNetT \\ Institute for Geophysics and Planetary Physics, Lawrence \\ Livermore National Laboratory, Livermore, CA 94550 \\ and \\ Sun Hong RHIE \\ Center for Particle Astrophysics, University of California, Berkeley, CA 94720
}

\begin{abstract}
We report on a calculation of large scale anisotropy in the cosmic microwave background radiation in the global monopole and texture models for cosmic structure formation. We have evolved the six component linear gravitational field along with the monopole or texture scalar fields numerically in an expanding universe and performed the Sachs-Wolfe integrals directly on the calculated gravitational fields. On scales $>7^{\circ}$, we find a Gaussian distribution with an approximately scale invariant fluctuation spectrum. The $\Delta T / T$ amplitude is a factor of 4-5 larger than the prediction of the standard CDM model with the same Hubble constant and density fluctuation normalization. The recently reported COBE-DMR results imply that global monopole and texture models require high bias factors or a large Hubble constant in contrast to standard CDM which requires very low $H_{0}$ and bias values. For $H_{0}=70 \frac{\mathrm{km}}{\mathrm{sec}} \mathrm{Mpc}^{-1}$, we find that normalizing to the COBE results implies $b_{8} \simeq 3.2 \pm 1.4$ (95\% c.l.). If we restrict ourselves to the range of bias factors thought to be reasonable before the announcement of the COBE results, $1.5 \lesssim b_{8} \lesssim 2.5$, then it is fair to conclude that global monopoles and textures are consistent with the COBE results and are a better fit than Standard CDM.
\end{abstract}

Submitted to The Astrophysical Journal Letters 
Topological defects which formed in a phase transition in the early universe provide an attractive mechanism for the generation of density perturbations which can grow to form galaxies and large scale structure. Cosmic strings (Vilenkin, 1980, Zel'dovich, 1980), global monopoles (Bariola and Vilenkin, 1989, Bennett and Rhie, 1990), and global textures (Turok, 1989) have all been proposed as possible seeds for cosmic structure formation. These theories are characterized by a single adjustable parameter, the Grand Unified theory symmetry breaking scale, $v$, and the value $\left(v \sim 10^{16} \mathrm{GeV}, G v^{2} \sim 10^{-6}\right)$ predicted by particle physics (Amaldi et al., 1991) also gives the correct amplitude to generate galaxies and large scale structure. In contrast, inflationary models generally cannot produce a reasonable perturbation amplitude without a rather extreme fine tuning of the coupling constant $\left(\lambda \sim 10^{-12}\right)$.

Recent studies (Cen et al., 1991, Park et al., 1991, Gooding et al., 1991 and 1992, and Turok and Spergel, 1990) of texture seeded density perturbations in a universe dominated by cold dark matter $(\mathrm{CDM})$ have indicated that this theory may solve two of the perceived problems with the standard CDM model: the lack of sufficient large scale structure and quasars at high redshift. Their results generally agree with our calculations of global monopole (GM) and texture (T) seeded structure formation (Bennett, Rhie, and Weinberg, 1992), but we also find that these nongaussian seeds tend to generate large galaxy pair velocities and cluster velocity dispersions (see also Bartlett, Gooding and Spergel, 1992). This can be alleviated by selecting a larger "bias" factor (i.e., a lower normalization of the density field).

Another major difference between the nongaussian GM \& T models and the gaussian inflationary models can be seen in the cosmic microwave background radiation (CMB) anisotropies. In inflation scenarios, $\Delta T / T$ is due to remnant quantum fluctuations crossing the horizon at last scattering, and analytic results for $\Delta T / T$ have been obtained for both the scalar mode responsible for the growth of cosmic structure (Bond and Efstathiou, 1987) and the tensor modes (gravity waves) (Abbott and Wise, 1984). For the standard exponential inflation models, only the scalar growing mode is important. With topological defects, metric fluctuations are generated by the relativistic dynamics of the defects inside the horizon, and $\Delta T / T$ is affected by the fluctuations in all six independent components of the gravitational field. Thus, we expect the topological seed models to predict larger $\Delta T / T$ for a fixed amplitude of density fluctuations. The recent detection of CMB anisotropy by the COBE DMR experiment (Smoot, et al., 1992) at a level somewhat higher than the prediction of the Standard CDM model should be regarded as encouraging for topological seed models. (Some non-standard inflationary models may also have significant tensor mode perturbations (Davis, et al., 1992), but they are certainly not the only theory with tensor modes.) 
A previous estimation of $\Delta T / T$ for the texture model (Turok and Spergel, 1990) was based on a simple analytic model of a single texture evolution. They found a non-Gaussian distribution of hot and cold spots at a level that seems to conflict with the COBE data. In this paper, we present realistic numerical calculations of $\Delta T / T$ on COBE scales in the GM\&T models with no simplifying assumptions.

$\Delta T / T$ on COBE scales reflects the variations in time delay (frequency shift) along the photon paths from last scattering until the present. This is the generalized Sachs-Wolfe effect (Sachs and Wolfe, 1967) where not only scalar growing mode but all gravitational field components contribute to the temperature fluctuations. If we choose a coordinate system such that the metric is $g_{\mu \nu}(x)=a(\eta)^{2}\left[\eta_{\mu \nu}+h_{\mu \nu}(x)\right]$, where $\eta_{\mu \nu}=\operatorname{diag}(-,+,+,+)$ and $h_{\mu \nu}$ is the metric perturbation, and choose a gauge $h_{0 \nu}=0$ (Veeraraghavan and Stebbins, 1990), then the temperature fluctuation is given by

$$
\frac{\Delta T}{T}=-\frac{1}{2} \int d \eta \hat{x}_{i} \hat{x}_{j} \frac{\partial h_{i j}}{\partial \eta},
$$

where $\hat{x}_{i}$ is the normal vector along the line of sight. Because GM\&T predict the early formation of objects such as quasars, we assume the universe was reionized at high redshift and take this into account by introducing a visibility function,

$$
f(z)=e^{h \Omega_{b}\left(1-(1+z)^{3 / 2}\right) / 21.7}
$$

which measures the fraction of photons present at redshift $z$ that will reach $z=0$ without undergoing Compton scattering. If we assume that the electrons which scatter each photon see $\langle\Delta T / T\rangle=0$, then we can account for reionization by inserting $f(z)$ inside the "Sachs-Wolfe" integral, eq. (1). This means that our

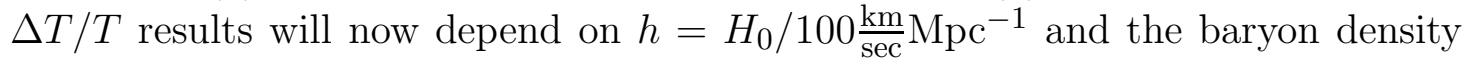
$\Omega_{b}$. We have done most of our calculations for $h \Omega_{b}=0.1$ which is about the largest plausible value. For $h \Omega_{b}=0.04, \Delta T / T$ is only $3 \%$ larger on COBE scales.

We evolve the source fields according to the field equations of motion in the Freedman-Robertson-Walker background and calculate the metric perturbations due to the energy-momentum of the scalar field by solving the linearized Einstein equations. The field equations are

$$
\ddot{\phi}^{p}+2 \frac{\dot{a}}{a} \dot{\phi}^{p}-\nabla^{2} \phi^{p}+a^{2} \lambda\left(\phi^{2}-v^{2}\right) \phi^{p}=0
$$

where $v$ is the vacuum expectation value of the field, and $p$ runs from 1-3 for monopoles and 1-4 for textures. Because the defect core size is very much smaller 
than the our grid spacing, we can use the equation for the $\lambda \rightarrow \infty$ and improve the dynamic range of the calculations (Bennett and Rhie, 1990),

$$
\left(\delta_{p q}-\frac{\phi^{p} \phi^{q}}{v^{2}}\right)\left(\ddot{\phi}^{q}+2 \frac{\dot{a}}{a} \dot{\phi}^{q}-\nabla^{2} \phi^{q}\right)=0 .
$$

The energy-momentum tensor for the scalar fields is given by

$$
\begin{aligned}
\Theta_{00} & =\frac{1}{2} \dot{\vec{\phi}}^{2}+\frac{1}{2}(\nabla \vec{\phi})^{2}, \\
\Theta_{0 i} & =\dot{\vec{\phi}} \cdot \partial_{i} \vec{\phi} \\
\Theta_{i j} & =\partial_{i} \vec{\phi} \cdot \partial_{j} \vec{\phi}+\frac{1}{2} \delta_{i j}\left(\frac{1}{2} \dot{\vec{\phi}}^{2}-\frac{1}{2}(\nabla \vec{\phi})^{2}\right),
\end{aligned}
$$

and the linearized Einstein equations are (Veeraraghavan and Stebbins, 1990)

$$
\begin{gathered}
\ddot{h}+\frac{\dot{a}}{a} \dot{h}+3 \frac{\dot{a}^{2}}{a^{2}} \delta_{c}=-8 \pi\left(\Theta_{00}+\Theta\right) \\
\ddot{\tilde{h}}_{i j}-\nabla^{2} \tilde{h}_{i j}+2 \frac{\dot{a}}{a} \dot{\tilde{h}}_{i j}-\frac{1}{3} \partial_{i} \partial_{j} h+\frac{1}{9} \delta_{i j} \nabla^{2} h \\
+\partial_{i} \partial_{k} \tilde{h}_{j k}+\partial_{j} \partial_{k} \tilde{h}_{i k}-\frac{2}{3} \delta_{i j} \partial_{k} \partial_{l} \tilde{h}_{k l}=16 \pi \tilde{\Theta}_{i j}
\end{gathered}
$$

where $h$ and $\Theta$ refer to the traces of $h_{i j}$ and $\Theta_{i j}$. The $\sim$ is used to refer to the trace-free terms $\tilde{h}_{i j}=h_{i j}-\frac{1}{3} \delta_{i j} h$ and $\tilde{\Theta}_{i j}=\Theta_{i j}-\frac{1}{3} \delta_{i j} \Theta$. The perturbation in cold dark matter $\delta_{c}$ obeys

$$
\dot{\delta}_{c}=-\frac{1}{2} \dot{h}
$$

and the following (non-dynamical) constraint equations must be satisfied,

$$
\begin{aligned}
& \frac{1}{2} \partial_{i} \partial_{j} \tilde{h}_{i j}+\frac{1}{3} \nabla^{2} h=8 \pi \Theta_{00}+3\left(\frac{\dot{a}}{a}\right)^{2} \delta_{c}-\frac{\dot{a}}{a} \dot{h}, \\
& \frac{1}{2} \partial_{j} \dot{\tilde{h}}_{i j}-\frac{1}{3} \partial_{i} \dot{h}=8 \pi \Theta_{0 i} .
\end{aligned}
$$

In theories with "external" sources such as global monopoles or textures, these equations impose important constraints on the initial conditions. In particular, since $\Theta_{00}$ cannot vanish if we are to have interesting density perturbations, the initial energy density fluctuations in the $\vec{\phi}$ field must be "compensated" by fluctuations of the opposite sign in the other fields. For the calculations reported in this 
paper, we have taken $h\left(\eta_{0}\right)=0, \dot{h}\left(\eta_{0}\right)=0, \tilde{h}_{i j}\left(\eta_{0}\right)=0, \dot{\tilde{h}}_{i j}\left(\eta_{0}\right)=0, \dot{\vec{\phi}}\left(\eta_{0}\right)=0$, and $\delta_{c}\left(\eta_{0}\right)=-(8 \pi / 3)(a / \dot{a})^{2} \Theta_{00}\left(\eta_{0}\right)$. Once the constraint equations, (9), are satisfied initially, they will be satisfied at subsequent times if the equations of motion, (6)-(8), are satisfied. Extreme care must be taken when evolving these equations numerically, because small numerical errors can excite growing mode solutions on scales outside the horizon where the physical modes do not grow. When these scales finally come inside the horizon, the errors can have grown large enough to compete with the physical perturbations that are generated by the source inside the horizon. We have found that we can keep these errors under control even when $\vec{\phi}$ takes random values on the scale of 1 grid spacing by going to extremely small time-steps $(\Delta t \approx 0.01 \Delta x)$. In order to satisfy the second equation in $(9)$, we find that global monopole and texture fields must be sufficiently smooth, and this limits us to an initial horizon size of $\gtrsim 8$ grid spacings.

Another difficulty with evolving eqs. (6)-(8) numerically is that the mixed partial derivatives in (7) make it difficult to come up with an explicit differencing scheme that is stable. Implicit differencing schemes would be prohibitively expensive in computer time because we would still have to take very small time-steps to avoid the super-horizon scale growing mode solution discussed above since the growing mode is a physical mode, not a purely numerical one.

Fortunately, we have found it possible to remove the offensive mixed partial into (7). This substitution yields

$$
\begin{aligned}
\ddot{\tilde{h}}_{i j} & =\nabla^{2} \tilde{h}_{i j}-2 \frac{\dot{a}}{a} \dot{\tilde{h}}_{i j}-\partial_{i} \partial_{j} h+\left.16 \pi \partial_{i} \vec{\phi} \cdot \partial_{j} \vec{\phi}\right|_{\eta=\eta_{0}}-32 \pi \int_{\eta_{0}}^{\eta} d \eta \dot{\vec{\phi}} \cdot \partial_{i} \partial_{j} \vec{\phi} \\
& +\delta_{i j}\left[\frac{1}{3} \nabla^{2} h+\frac{16 \pi}{3} \overrightarrow{\dot{\phi}^{2}}+4\left(\frac{\dot{a}}{a}\right)^{2} \delta_{c}-\frac{4}{3} \frac{\dot{a}}{a} \dot{h}\right],
\end{aligned}
$$

for eq. (7). The mixed partial derivatives of $h$ and $\vec{\phi}$ in eq. (10) do not give rise to numerical instabilities because the equations of motion for $h$ and $\vec{\phi}((6)$ and (4)) do not contain any mixed partial derivatives.

Our numerical simulations use a modified second order leapfrog scheme to evolve eqns. (4), (6), (8), and (10) in time. $\Delta T / T$ is determined by integrating eq. (1) (with the visibility function (2) inserted) along photon trajectories that converge to a point at the end of the simulation. We have done about 25 simulations on $64^{3}$ grids and 12 simulations on $100^{3}$ grids for each of the global monopole and texture models. The RMS $\Delta T / T$ values for the $100^{3}$ grids are $17.80 \pm 2.00 G v_{m}^{2}$ and $10.29 \pm 1.43 G v_{t}^{2}$ for monopoles and textures respectively. $\left(v_{m}\right.$ and $v_{t}$ refer to the 
vacuum expectation values of the monopole and texture fields.) These numbers can be compared to the RMS fluctuation measured by COBE $(\Delta T / T)_{R M S}=$ $1.10 \pm 0.18 \times 10^{-5}$ to yield $G v_{m}^{2}=6.18 \pm 1.23 \times 10^{-7}$ for global monopoles or $G v_{t}^{2}=1.07 \pm 0.23 \times 10^{-6}$ for textures if we assume that monopoles or textures are responsible for the $\Delta T / T$ observed by COBE.

Figs. 1 and 2 show the simulated full-sky temperature maps for the global monopole and texture models respectively smoothed to the same $10^{\circ}$ scale as the COBE maps. The scale on these plots is given in terms of $G v^{2}$. Figs. 4 and 5 show histograms of the $\Delta T / T$ distributions in the monopole and texture cases. Note that after the smoothing the maps contain only about 400 independent pixels. Thus, the departures from a Gaussian distribution are not significant.

Fig. 3 shows the angular power spectrum, $\Delta T_{l}^{2}$, of our simulated $\Delta T / T$ maps.

$$
\begin{gathered}
\frac{\Delta T}{T}(\theta, \phi)=\sum_{l, m} a_{l m} Y_{l m}(\theta, \phi), \\
\Delta T_{l}^{2}=\frac{1}{4 \pi} \sum_{m}\left|a_{l m}\right|^{2} .
\end{gathered}
$$

The solid and dashed curves give the best fit to the predicted power spectrum for Harrison-Zel'dovich $(n=1)$ primordial adiabatic density perturbations (Bond and Efstathiou, 1987),

$$
\Delta T_{l}^{2}=\left(Q_{r m s-P S}\right)^{2} \frac{(2 l+1)}{5} \frac{\Gamma(l+(n-1) / 2) \Gamma((9-n) / 2)}{\Gamma(l+(5-n) / 2) \Gamma((3+n) / 2)} .
$$

The best fit of the form, (13), gives $n=1.1 \pm 0.3$ for global monopoles and $n=1.2 \pm 0.2$ for textures when we remove the quadrupole from the fit as was done for the COBE data. (The error bars in these fits reflect mainly systematic errors.) The Harrison-Zel'dovich value, $n=1$, gives a good fit to our simulations and to the COBE data, so it makes sense to compare the fit amplitudes. The COBE value is $Q_{r m s-P S}=6.11 \pm 1.46 \times 10^{-6}$, and we obtain $Q_{r m s-P S}=8.7 \pm 1.6 G v_{m}^{2}$ for monopoles and $Q_{r m s-P S}=4.7 \pm 0.5 G v_{t}^{2}$ for textures. A comparison of these values gives $G v_{m}^{2}=7.0 \pm 2.1 \times 10^{-7}$ and $G v_{t}^{2}=1.30 \pm 0.34 \times 10^{-6}$ consistent with the values obtained above.

It is worth noting that topological defect theories generically predict $\Delta T / T$ and $\delta \rho / \rho$ spectra that are slightly steeper than Harrison-Zel'dovich on very large scales $(\gtrsim 1000 \mathrm{Mpc})$. The reason for this is that with topological defects, unlike inflation, the gravitational fields are generated inside the horizon, so that scales 
near the horizon have yet to receive their full "share" of perturbations. Thus, the largest scales are expected to have less power than the scale-free spectrum (i.e. $n>1$ ). (This effect is partially compensated for by the effects of reionization which reduce $\Delta T / T$ on small scales.) For cosmic strings, the effect should be even more pronounced since the coherence scale of the strings is smaller than that of global monopoles or textures (Bennett, Stebbins, and Bouchet, 1992). In contrast, power law inflationary models (Davis, et al., 1992) which are able to fit the COBE results with a reasonable value for $b_{8}$ predict $n<1$. Thus, if the four year COBE results converge to $n=1.50 \pm 0.25$, it will be fair to conclude that topological defect models are preferred over quantum fluctuations during inflation as the source of the primordial density perturbations.

\begin{tabular}{|c|c|c|c|c|}
\hline & \multicolumn{2}{|c|}{ Monopoles } & \multicolumn{2}{c|}{ Textures } \\
\hline$h$ & $G v_{m}^{2}$ & $b_{8}$ & $G v_{t}^{2}$ & $b_{8}$ \\
\hline 0.5 & $2.49 \times 10^{-6} / b_{8}$ & $4.03 \pm 0.80$ & $4.56 \times 10^{-6} / b_{8}$ & $4.27 \pm 0.98$ \\
0.6 & $2.19 \times 10^{-6} / b_{8}$ & $3.54 \pm 0.70$ & $3.93 \times 10^{-6} / b_{8}$ & $3.68 \pm 0.84$ \\
0.7 & $1.96 \times 10^{-6} / b_{8}$ & $3.17 \pm 0.63$ & $3.48 \times 10^{-6} / b_{8}$ & $3.25 \pm 0.75$ \\
0.8 & $1.82 \times 10^{-6} / b_{8}$ & $2.94 \pm 0.58$ & $3.17 \times 10^{-6} / b_{8}$ & $2.97 \pm 0.68$ \\
1.0 & $1.64 \times 10^{-6} / b_{8}$ & $2.65 \pm 0.53$ & $2.75 \times 10^{-6} / b_{8}$ & $2.57 \pm 0.59$ \\
\hline
\end{tabular}

Table 1. The scalar field normalizations and best fit bias parameters, $b_{8}$, to the COBE-DMR RMS anisotropy at $10^{\circ}$ are tabulated as a function of $h .1 \sigma$ errors are reported.

In order to translate our results into limits on theories of cosmic structure formation, we must normalize $G v^{2}$ to give a reasonable spectrum of density perturbations. Because of uncertainties in the relationship between the number density of galaxies and the mass density, this normalization is conventionally given in terms of a bias factor, $b_{8}$, such that the RMS mass density fluctuation $=1 / b_{8}$ after smoothing with an $8 h^{-1} \mathrm{Mpc}$ radius top hat. Table 1 gives these normalizations as determined in Bennett, Rhie, and Weinberg (1992).

Table 1 also lists the predicted $b_{8}$ values as a function of $h=H_{0} / 100 \frac{\mathrm{km}}{\mathrm{sec}} \mathrm{Mpc}^{-1}$ as determined by a comparisons of the predicted RMS fluctuation at $10^{\circ}$. We can see that the predicted $b_{8}$ values run very high for small values of the Hubble constant. Choosing a large Hubble constant in the $\Omega=1$ universe that we have assumed is problematic due to the implied very short age for the universe, but with $h=0.7$ (the smallest value that is consistent with most of the measured values of $h$ ) $b_{8}=2.5$ is within $\sim 1 \sigma$ of the mean in the both the global monopole and 
texture models. Thus, global monopoles and textures with a bias factor in the range of $2.5-3$ seem to be in reasonably good agreement with the COBE data. In a separate study of large scale structure in the global monopole and texture models, (Bennett, Rhie, and Weinberg, 1992), we find that these high bias global monopole and texture models do reasonably well in matching the observed large scale structure. Further work is required to see if the required biasing can be obtained dynamically, however.

Finally, let us compare our results to those of other, well motivated theories of large scale structure formation. We find that for similar values of $h$ and $b_{8}$, global monopole and textures predict $\Delta T / T$ on COBE scales that is a factor of 4-5 larger than the standard CDM prediction (Bond and Efstathiou, 1987). With a reasonable bias value, $1.5 \gtrsim b_{8} \gtrsim 2.5$, this model is inconsistent with the COBE measurement for $h>0.5$ and but perhaps barely consistent for $h=0.5$. If we demand that $1.5 \gtrsim b_{8} \gtrsim 2.5$ for global monopoles and textures, we find consistency with COBE for the entire range, $0.5<h<1.0$ at the $2 \sigma$ confidence level and $0.7<h<1.0$ at the $1 \sigma$ level. The power law inflationary models discussed by Davis, et al., (1992) can be made consistent with reasonable $b_{8}$ values because they have significant contributions to $\Delta T / T$ from tensor modes that do not contribute to $\delta \rho / \rho$. These models do seem to have some difficulty with forming galaxies early enough, however (Adams, et al., 1992). Other models in with a smaller amount of CDM, such as hot + cold DM models or low $\Omega$ models seem to fit the COBE results reasonably well (Wright, et al., 1992), but they are less attractive theoretically. Cosmic Strings + HDM seem to be a good fit to the COBE results (Bennett, Stebbins, and Bouchet, 1992), but the theoretical error bars are presently rather large. Thus, none of the best motivated models are singled out by the COBE results, but global monopoles and textures are arguably the best fit to COBE among the $\Omega=1$ pure CDM models. Further work into the details of galaxy formation and $\Delta T / T$ on smaller angular scales is certainly warranted.

Acknowledgements: We would like to thank A. Stebbins and D. Weinberg for helpful discussions. This work was supported in part the U.S. Department of Energy at the Lawrence Livermore National Laboratory under contract No. W-7405-Eng-48 and by the NSF grant No. PHY-9109414. 


\section{REFERENCES}

Abbott, L., and Wise, M., 1984, Nucl. Phys. B 244, 541.

Adams, F. C., Bond, J. R., Freese, K., Frieman, J. A., and Olinto, A. V., 1992, CITA preprint.

Amaldi, U. et al., 1991, Phys. Lett. B 260, 447.

Bartlett, J., Gooding, A. K., and Spergel, D. N., 1992, Berkeley preprint.

Bariola, M., and Vilenkin, A., 1989, Phys. Rev. Lett. 63, 341.

Bennett, D. P., Stebbins, A., and Bouchet, F. R., 1992, IGPP preprint UCRL-JC110803, submitted to Astrophys. J. Lett.

Bennett, D. P., and Rhie, S. H., 1990, Phys. Rev. Lett. 65, 1709.

Bennett, D. P., Rhie, S. H., and Weinberg, D. H. 1992, in preparation.

Bond, J. R., and Efstathiou, 1987, Mon. Not. R. Astron. Soc. 226, 655.

Cen, R. Y., Ostriker, J. P., Spergel, D. N., and Turok, N., 1991, Astrophys. J., 383, 1.

Davis, R., Hodges, H., Smoot, G. F., Steinhardt, P. J., and Turner, M. S., 1992, preprint.

Gooding, A. K., Spergel, D. N., and Turok, N., 1991, Astrophys. J., 372, L5.

Gooding, A. K., et al., 1992, Astrophys. J., 393, 42.

Park, C., Spergel, D. N., and Turok, N., 1991, Astrophys. J., 372, L53.

Sachs, K., and Wolfe, A. M., 1967, Astrophys. J., 147, 73.

Smoot, G., et al., 1992, COBE preprint.

Turok, N., 1989, Phys. Rev. Lett. 63, 2625.

Turok, N., and Spergel, D. N., 1990, Phys. Rev. Lett. 64, 2736.

Veeraraghavan, S., and Stebbins, A., 1990, Astrophys. J., 365, 37.

Vilenkin, A., 1980, Phys. Rev. Lett. 46, 1169, 1496(E).

Wright, E., et al., 1992, COBE preprint.

Zel'dovich, Y. B., 1980, Mon. Not. R. Astron. Soc. 192, 663. 


\section{FIGURE CAPTIONS}

1) A $\Delta T / T$ map for the global monopole model generated by our global field evolution code is displayed in a full-sky equal area projection. The scale is given in units of $G v_{m}^{2}$.

2) A $\Delta T / T$ map for the texture model generated by our global field evolution code is displayed in a full-sky equal area projection. The scale is given in units of $G v_{t}^{2}$.

3) The average $\Delta T / T$ power spectrum is plotted for $12100^{3}$ monopole simulations and $12100^{3}$ texture simulations. The solid and dashed curves give the best fit to the power spectrum derived for a Harrison-Zel'dovich spectrum of primordial adiabatic density perturbations. The error bars give the RMS deviation from the mean, so they reflect the expected deviation for a single realization.

4) A histogram of the pixels in convolved $\Delta T / T$ map shown in Fig. 1. The bins on the edges of the histogram include all the points outside the limits of the figure. The dashed curve is the histogram for a Gaussian with the same RMS.

5) A histogram of the pixels in convolved $\Delta T / T$ map shown in Fig. 2. The bins on the edges of the histogram include all the points outside the limits of the figure. The dashed curve is the histogram for a Gaussian with the same RMS. 